\title{
ACUTE MEGAKARYOCYTIC LEUKEMIA (FAB M7)
}

\author{
ARA $T^{1}$, ZAMAN MK $^{2}$, AFROSE $\mathrm{S}^{3}$, ISLAM MS ${ }^{4}$, BISWAS AR ${ }^{5}, \mathrm{KHAN} \mathrm{MA}^{6}$, RAHMAN MM ${ }^{7}$
}

\begin{abstract}
:
Acute megakaryocytic leukemia (AML M7) is a rare type of acute leukemia often presented with myelofibrosis. This report describes a 65 years old female who presented with progressive weakness and fatigue. She was diagnosed as a case of de novo AMLM-7 on the basis of peripheral blood finding, bone marrow examination report and immunophenotyping. She refused chemotherapy, received only supportive therapy and died after two months of diagnosis.
\end{abstract}

Key Words: Acute megakaryocytic leukemia, thrombocytosis, immunophenotyping.

J Dhaka Med Coll. 2011; 20(1) : 89-92.

\section{Introduction:}

Acute myeloid leukemia (AML), and acute nonlymphocytic leukemia (ANLL) refer to a group of marrow based neoplasm that have clinical similarities and distinct morphologic, immunophenotypic, cytogenetic and molecular features. The malignant cell in AML is a blast that most often shows myeloid or monocytic differentiation. In few patients, blasts have erythroid or megakaryocytic differentiation ${ }^{1}$. Myeloid lineage is demonstrated by morphology (i.e. Auer rods), cytochemical staining (i.e. MPO, Sudan black, chloroacetate esterase, nonspecific esterase etc.), and or immunophenotyping ( $\mathrm{CD}$ markers). The FAB Classification identified eight subtypes of AML based on morphology and cytochemical staining with immunophenotypic data in some instances. Four types (M0, M1, M2, and M3) are predominantly granulocytic and differ according to the extent of maturation. M4 is both granulocytic and monocytic, with at least $20 \%$ monocytic cells, whereas M5 is predominantly monocytic (at least 50\% monocytic cells). M6 shows primarily erythroid differentiation with dysplastic features including megaloblastic changes, and M7 is acute megakaryocytic leukemia (AML-M7) identified by the presence of megakaryocytic antigens demonstrated by flow cytometry or immunohistochemistry or the presence of platelet peroxides or a demarcation membrane system on ultrastuctural studies by electron microscopy ${ }^{2}$.

\section{Case Report:}

A 65 years old female attended a district level hospital with three month history of gradually increasing weakness and breathlessness. Complete blood count with peripheral blood film revealed severe anaemia, very high ESR and leuco-erythroblastic blood picture with gross thrombocytosis. She was treated conservatively with three units of whole blood and referred to a specialized haematology centre for proper diagnosis and treatment. But she left hospital, remained clinically stable at home for about 6 months. As her symptoms got aggravated she visited the local hospital and was transferred to our hospital with features of anaemia (gradually increasing weakness, shortness of breath on exertion, palpitation). On examination marked pallor and bilateral mild ankle edema was noted. She had no bony tenderness, lymphadenopathy or organomegaly. Other systems were normal. On investigation: Complete blood count with

1. Dr.Tasneem Ara, Assistant Professor, Dept. of Hematology, Dhaka Medical College and Hospital, Dhaka.

2. Dr. Muhammad Kamruzzaman, Medical Officer, Dept. of Hematology, Dhaka Medical College and Hospital, Dhaka.

3. Dr. Salma Afrose, Associate Professor, Dept. of Hematology, Dhaka Medical College and Hospital, Dhaka..

4. Dr. M Sirajul Islam, Assistant Professor, Dept. of Hematology, Dhaka Medical College and Hospital, Dhaka.

5. Dr. Akhil Ranjon Biswas, Registrar Dept. of Hematology, Dhaka Medical College and Hospital, Dhaka.

6. Dr. Mohiuddin Ahmed Khan, Professor, Dept. of Hematology, Dhaka Medical College and Hospital, Dhaka.

7. Dr. M Mizanur Rahman, Registrar, Dept. of Hematology, Dhaka Medical College and Hospital, Dhaka.

Correspondence: Dr. Tasneem Ara, Assistant Professor, Department of Hematology, Dhaka Medical College, Dhaka, Bangladesh, E-mail: aratasneem@ymail.com. 
peripheral blood revealed hemoglobin $5 \mathrm{~g} / \mathrm{dl}$, leucocyte count $29 \times 10^{9} /$ Lwith a differential count of $-30 \%$ segmented neutrophils, $20 \%$ lymphocytes, 03\% eosinophils and 47\% blast and platelet count of $1200 \times 10^{9} /$ L.Peripheral blood smear examination showed normocytic normochromic red blood cells including few nucleated red blood cells, white blood cells showed left, shift with significant number of undifferentiated blast suggestive of acute leukemia. Platelets were markedly increased with platelet anisocytosis including many giant platelets (Figure-1). Bone marrow aspiration was difficult; blood tap was obtained with sufficient cells in marrow blood smear. The cells were medium to large sized blast having basophilic agranular cytoplasm dense chromatin, 1-3 nucleoli including few cells showing cytoplasmic blebs (Figure - 2).

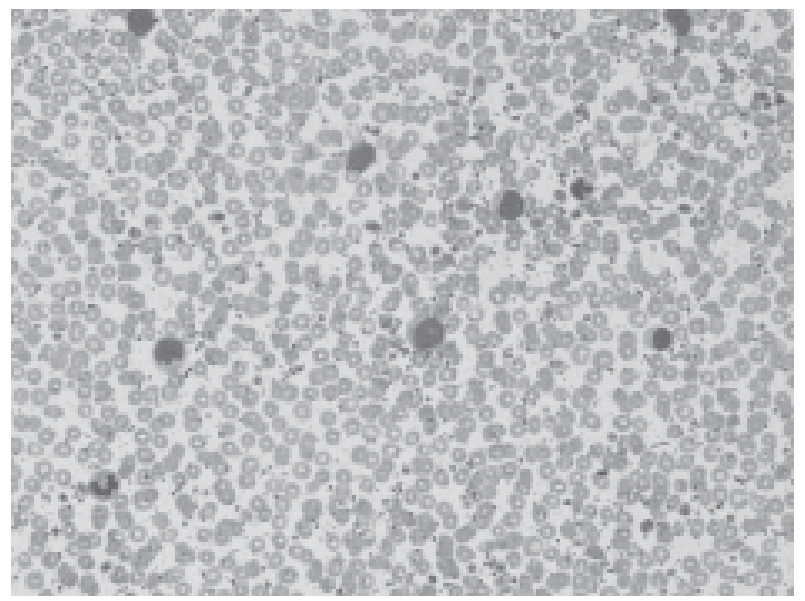

Fig.-1: Peripheral blood film shows blasts and a giant platelet

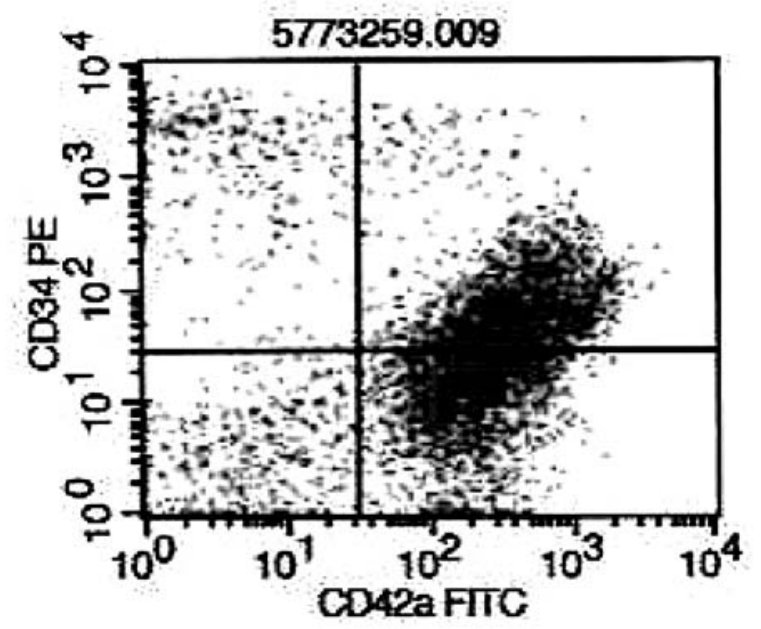

Immunophenotype: T-cell markers: CD3, CD5, CD7 were negative, B cell markers: CD19, CD22 were negative, myeloid associated markers: CD13, CD33 and platelet associated markers CD42a, CD61 were positive (Figure-3), CD14, CD15, CD117, MPO were negative, other markers: CD45, CD34, HLA-DR are positive, CD10 was negative. The biochemical parameters including electrolytes, uric acid, creatinine, bilirubin, liver enzymes were normal. Serum LDH was slightly raised. The diagnosis was confirmed as AML-M7 as the blasts were positive for CD42 and CD61 (megakaryocyte specific antigen) along with myeloid markers CD13 and CD33. Other investigation reports were normal including skeletal survey for bony lytic lesions. We explained treatment options but our patient refused chemotherapy and died two months after diagnosis.

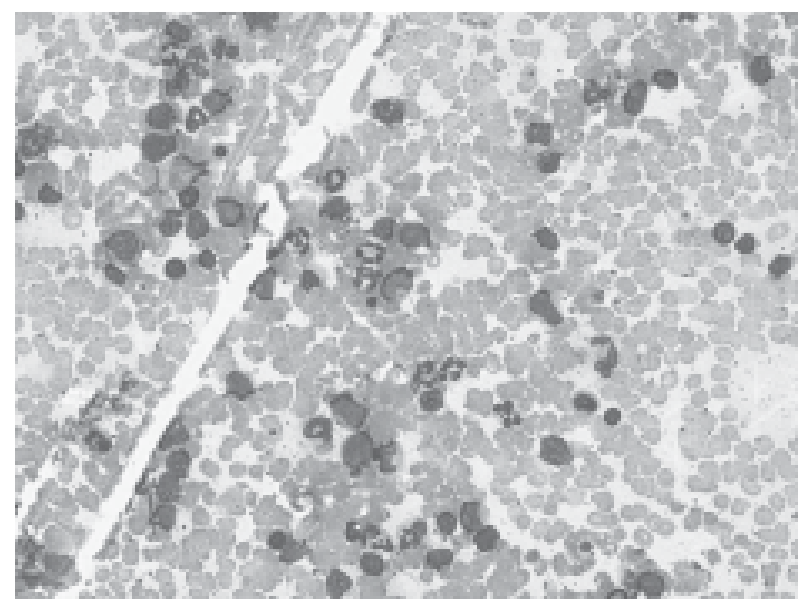

Fig.-2: Bone marrow smear shows plenty undifferentiated blast

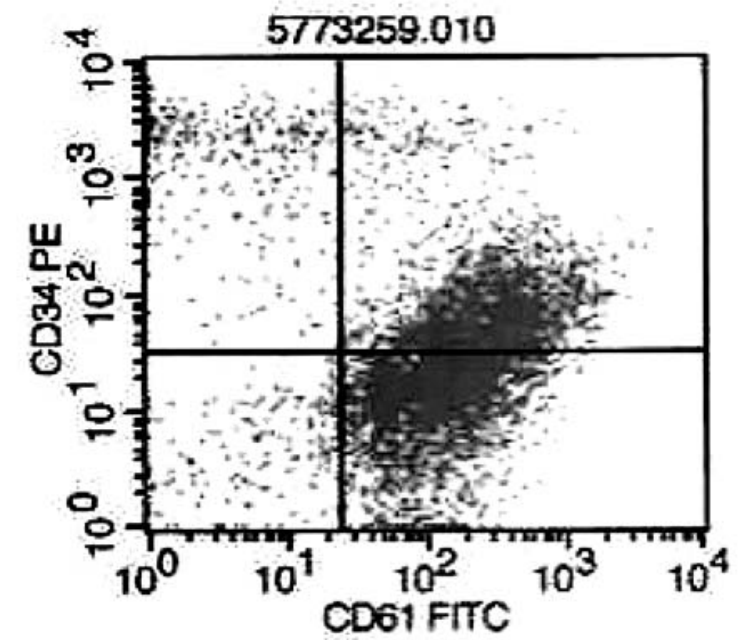

Fig.-3: Immunophenotyping shows strong positivity for CD42a (left panel) and CD61 (right panel). 


\section{Discussion:}

The incidence of acute leukemia is approximately 2.3 per 100000 people per year.AML M-7 is a rare subtype of leukemia and represents $1.2 \%$ of cases of adult leukemia, compared to $3-10 \%$ of childhood leukemia ${ }^{3}$. Acute megakaryocytic leukaemia is a form of leukaemia where a majority of the blasts are magakaryoblastic. It is classified under M-7 in the French-American-British classification. There is bimodal age distribution, with one peak in the infancy another in the elderly 4 .Our patient was a sixty five years elderly female. Clinical features are not different from other type of AML but organomegaly is noted infrequently in adults. In our patient symptoms of anemia that is progressive weakness and mild ankle edema were only symptoms. Cytopenias are usually present but $30 \%$ of patients have platelet counts $>100000 / \mathrm{uL}$ as in our case who had very high platelet count (1200x109/L). Osteosclerotic and osteolytic lesions have been described in few case reports 5, 6, 7. The diagnosis depends on the expression of at least one platelet antigen (CD41, CD42b, CD61 or factor V111 related antigen) on the leukemic cells ${ }^{8,9}$. In our patient Immunophenotyping of peripheral blood revealed blast cells were positive for $\mathrm{CD} 41$ and CD 61. The bone marrow may be difficult to aspirate, and more than two third of patients have significant fibrosis ${ }^{10}$. In or case; repeated attempts at aspiration were unsuccessful, finally blood tap was obtained and diagnosis was made from the cell trail. Unfortunately we could not perform trephine biopsy including reticulin stain to find out the evidence of fibrosis. AML M 7 may present as de novo leukemia, secondary leukemia after chemotherapy, or transformed myeloproliferative disorders and myelodysplastic syndromes ${ }^{11}$. Our report describes a de novo presentation of AML M7 with thrombocytosis. Although nonspecific, cytogenetic abnormalities are more frequent $(>90 \%)$ in AMLM-7 than in other subtypes of

AML ${ }^{8}$. Cytogenetic analysis was not carried out in our case. The prognosis of adult AML M7 is usually poor when compared to other AML types. The complete remission (CR) rate for adult AMLM-7 is 43 to $50 \%$ and median disease free survival is only 6 to 10 months ${ }^{3}$. An ECOG series reported an inferior disease free survival in M7 AML compared to non M7 AML, and found M7 AML to be an independent adverse prognostic factor for DFS ${ }^{3}$. However complete remission and long term survival are common in children with AML M7 specially in children with Down syndrome ${ }^{12,13}$. Because of the poor prognosis of adult AMLM-7 early allogenic SCT should be considered. Interestingly our patient remained alive and clinically stable for several months with symptomatic treatment despite poor prognosis of AML-M7.

\section{Conclusion:}

The morphologic diagnosis of AML M-7 can be difficult and confused with L2 subtype of ALL or with AML M1. Cytoplasmic blebs suggestive of megakaryocytic differentiation may be present on the blasts. As AML-M7 represents a proliferation predominantly of megakaryoblasts it can be diagnosed by immunophenotyping demonstrating the expression of one or more platelet specific antigen along with myeloid markers. We conclude that acute megakaryocytic leukemia should be considered in the differential diagnosis of undifferentiated acute leukemia with high platelet count.

\section{References:}

1. Maria R. Baer. Jhon P. Green et al. Acute Myeloid Leukemia in Adult; Wintrobe, s Clinical Hematology; $12^{\text {th }}, 2009:$ p.79; 1883, 1849, 1852, 1858.

2. Werdlow SH, Campoo E, Harris NL,Jaffe ES, PilerisA, Setin H, et.al., editors. WHO classification of tumors of haemopoietic and lymphoid tissues. $4^{\text {th }}$ Edition. Lyon:JARK Press;2008.

3. Talman MS, NeubergD, Cotelingam JD, etal. Acute megakaryocytic leukaemia:The Eastern Coperative Oncology Group experience. Blood 2000:65:5-13

4. Peterson BA,Levine EG. Uncommon subtypes of acute nonlymphocytic leukemia: clinical features and management of FABM5, M6, and M7.Semin Oncol 1987; 14: 425-434.

5. Moody A, Simpson E, Shaw D, Florid radiological appearance of megakaryoblastic leukaemia an aid to diagnosis. Pediatr Radiology 1989;486-488.

6. Muler JH, ValdezR,Hayes C,Kaminiski MS. Acute megakaryocytic leukaemia presenting as hypercalcemia with skeletal lytic lesion.Eur J Hematol 2002;68: 392-396. 
7. Kumar S,Mow B, Kaufman S. Hypercalcemia complicating leukemic transformation of agnogenic myeloid metaplasia. $M$ ayo clinic Proc 1999;74:1233-1237.

8. Khalidi HS, Medeiros LJ, Chang KL, et al. The immunophenotype of adult acute myeloid leukemia: high frequency of lymphoid antigen expression and comparison of immunophenotype, FrenchAmerican-British classification, and karyotypic abnormalities. Am J Clin Pathol 1998; 109:211220.

9. Bene MC, Castoldi G, Knapp W, et al. Proposals for the immunological classification of acute leukemias. European Group for the Immunological Characterization of Leukemias (EGIL). Leukemia 1995;9:1783-1786
10. Medical Research Council Leukaemia Committee. The relation between morphology and other features of acute myeloid leukemia, and their prognostic significance. Br J Haematol 1975; 31:165-180.

11. Cheson BD, Bennett JM, Kopecky KJ et al. Revised recommendations of the International Working Group for Diagnosis, Standardization of Response Criteria, Treatment Outcomes, and Reporting Standards for Therapeutic Trials in Acute Myeloid Leukemia. J Clin Oncol 2003; 21:4642-4649.

12. Hitzler, JK2007 Acute megakaryoblastic leukaemia in Down syndrome. Paeditric blood and cancer 49(7 suppl: 1066-9).

13. Hama A,Yagasaki H,Takahashi Y, et. al. Acute megakaryoblastic leukemia in children: a comparison of AMKL with or without Down syndrome 2008; Br. J. Haematol.140(5): 552-61. 